\title{
Anthocyanins from Black Chokeberry Delayed Ageing-related Degenerative Changes in the Heart
}

\author{
Dexin Xu, Jing Yang, Wenchen Yu, Jie Wei* \\ Department of Liaoning University; Chongshan Middle road 66, Huanggu District, Shenyang, CHINA.
}

\begin{abstract}
Aim: Aging is the greatest risk factor for most neurodegenerative diseases. Cardiovascular circulatory system is an important part of maintaining normal human life activities. The core of the aging process is DNA damage, a food additive called Anthocyanin which has shown high efficacy in preventing aging, We examine whether anthocyanins could keep young mice from accelerated ageing of the heart. Materials and Methods: In order to accelerate ageing, Mice of Kunming were injected with D-galactose and they were given anthocyanins of 20 and $40 \mathrm{mg} / \mathrm{kg}$ as an intervention. Results: The whole function and structure of heart were evaluated after eight weeks and the expression levels of genes involved in the DNA damage signalling pathway were evaluated through Western blot analysis. Reduction of the heart index and the heart tissue damage was delayed by Anthocyanins. it maintained the stability of the redox system in serum such as GSH-PX, T-SOD and MDA and heart tissue. In the DNA damage signalling pathway, the expression levels of sensors such as ATM and ATR and effectors such as Chk1 and Chk2 were reduced. Conclusion: Anthocyanins can be diffusely used to slow ageing-related deterioration of heart structure and function by inhibiting DNA damage in the field of health products.
\end{abstract}

Key words: Anthocyanins, Aornia mealnocarpa, Aged, DNA damage, Molecular docking.

\section{INTRODUCTION}

Maintenance of normal metabolic levels is critical for healthy adults; Now the heart ages are keeping a stable rate, but heart function could be decreased gradually. ${ }^{1-3}$ Even if some accessible therapies can improve symptoms, ageing-related heart injury is also irreversible and is accompanied by high rates of morbidity and mortality due to increased disease risk, including Senile ischemic cardiomyopathy, various cardiovascular and cerebrovascular diseases. ${ }^{4}$ it has been indicated that DNA damage plays a role in ageing, it is also found that it is correlative with strengthening in DNA damage in many different tissues of aged organism. ${ }^{5,6}$ Without properly functioning DNA damage response and repair pathways, proliferating stressors (such as injuries or transplants) could bring about increased levels of unrepaired DNA damage and missing of heart function. ${ }^{3-7}$ Many studies have showed that the core of anti-aging response is DNA damage responses. ${ }^{6,7}$ And the body antioxidant capacity and inflammation level is also an important part of aging mechanism.

Aronia melanocarpa Elliott, sometimes called black chokeberry, belongs to the Rosaceae family. The aronia are more and more popular than before because of its fructification having a high content of anthocyanins which have strong antioxidant activity. ${ }^{8}$ Aronia products are well received as nutritional supplements and the fructification can be added to juices, fruit tea, wines and jams, it also be used as a source of natural food colourants. In fact, black chokeberries possess the highest antioxidant activity among berries and other fruits investigated so far, as measured with the oxygen radical scavenging
Submission Date: 23-04-2018; Revision Date: 14-08-2018; Accepted Date: 23-10-2018

DOI: 10.5530/ijper.53.1.15 Correspondence: Dr. Jie Wei, Department of Life Science of Liaoning University; Chongshan Middle road 66, Huanggu District, Shenyang, CHINA.

Phone: +86-24-62202232 E-mail: J.Wei2015@hotmail. com

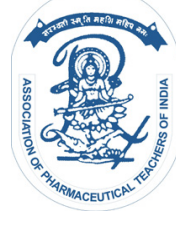

www.ijper.org 
capacity (ORAC) assay. The formation of free radicals is strongly associated with lipid peroxidation and has also been implicated in the development of all kinds of diseases, including the inflammation, the carcinogenesis and coronary heart disease, as well as cellular ageing. ${ }^{9}$

Lacking of proper function of DNA damage response, some injuries could bring about loss of heart function and increase levels of unrepaired DNA damage. So we should judge whether prophylactic anthocyanin treatments of aged mice could preserve youthful heart function and inhibit DNA damage accumulation and judge whether anthocyanin treatments were effective in slowing ageing-related degeneration of the heart. Our research can be the first to demonstrate a beneficial effect of anti-DNA damage with anthocyanin treatments on ageing heart. Anthocyanins in Aornia mealnocarpa will be a prospective source of anti-ageing agents that could have the potential to be used in health food products.

Owing to the aging population, more efficient ways are needed to be found to prevent or delay the eventual progression from aging. Based on previously reported anti-aging effects of anthocyanins, ${ }^{10}$ the current work was designed to identify and clarify effects from chokeberry through molecular dynamic simulations (MD) and animal text to investigate the potential mechanism associated with the anti-aging activity of these components.

\section{MATERIALS AND METHODS}

Anthocyanins in Aronia melanocarpa Elliot were extracted by the Biotechnology Innovation Lab of Liaoning University. The pure compounds including cyanidin-3-arabinoside $(4.7 \mathrm{mg} / \mathrm{g})$ and cyanidin-3-galactoside $(4.7 \mathrm{mg} / \mathrm{g})$, cyanidin-3-xyloside $(0.47 \mathrm{mg} / \mathrm{g})$ and cyanidin-3-glucoside $(0.1 \mathrm{mg} / \mathrm{g})$ were isolated from anthocyanins. D-galactose and tea polyphenols (TP) were purchased from Sangon Biotech Co., Ltd. (Shanghai, China).

\section{Molecular Docking and Molecular Dynamic (MD) Simulations Studies}

Molecular docking calculations were carried out to visualize the binding site of 2 anthocyanins to CDK5$\mathrm{p} 25$. The docking calculations were performed by using Autodock 4.2.5.1 Tools. Protein models were first modified by adding the all hydrogens and removing of water molecules using the builder module of Autodock. The 3D structure of 2 anthocyanins was downloaded from PUBCHEM-OPEN CHEMISTRY DATABASE (htEGCGs://pubchem.ncbi.nlm.nih.gov/substance). Both CDK5-p25 and 2 anthocyanins molecules were prepared using AutoDock Tools 1.5.6 before docking, The docking was carried out with $126 \times 126 \times 1260.375$ $\AA$ spacing grids covering the entire surface of CDK5-p25. The Lamarckian genetic algorithm, which is considered one of the most appropriate docking methods available in AutoDock, was used in the docking analysis.

Molecular docking results determine a general binding mode of ligand (2 anthocyanins). Nevertheless, MD simulation on the ligand-CDK5-p25 complex for further investigation of the effects of ligand binding on the conformation of protein was used. A MD simulation was performed using the AutoDockTools-1.5.6 software package. The structure and regulation of the CDK5-p25 (nck5a) complex (PDB entry 1H4L) was downloaded from the Protein Database (RCSB). The model of 2 anthocyanins (cyanidin 3-arabinoside and cyanidin 3-galactoside) monomer were constructed by using the Chem3D 16.0 software package.

\section{Treatments}

Adult male Kunming mice (about 8 weeks old) weighing about $35 \mathrm{~g}$ were provided by Changsheng Co., Ltd. (China). The medical animal license number is SCXK2015-0001. Sixty mice were divided into 4 experimental groups and a control group. In order to accelerate the ageing process, $150 \mathrm{mg} / \mathrm{kg} / \mathrm{d}$ D-galactose are used to inject into the experimental group mice. Experimental groups included Model group (treated with normal saline), a $20 \mathrm{mg} / \mathrm{kg}$ low-dose anthocyanin group (anthocyanins-20), a $40 \mathrm{mg} / \mathrm{kg}$ high-dose anthocyanin group (anthocyanins-40) and $20 \mathrm{mg} / \mathrm{kg}$ TP group. All drugs were administered by drinking water for 8 weeks. Body weight was recorded once a week, Changes in body weight have appeared in previous research on liver aging. ${ }^{11}$ After 8 weeks, the heart index was calculated (heart wet weight vs. body weight, $\mathrm{mg} / \mathrm{g}$ ).

\section{Histological Examination}

All parts of every mouse heart were used to prepare for paraffin sections and then stained with Masson's trichrome stain for light microscope visualization. Image-Pro Plus 6.0. Software was used to calculate the percent area of collagen deposition from each section.

\section{Western Blot Analysis}

The expression of ataxia telangiectasia mutated (ATM), ATM and Rad3-related protein (ATR), checkpoint kinase 1 (Chk1), checkpoint kinase 2(Chk2) were judged by Western blot analysis. We extract proteins from identical parts of mouse hearts. Protein samples of $100 \mu \mathrm{g}$ were separated through 10\% SDS-PAGE. Polyclonal rabbit anti-ATM, anti-ATR, anti-Chk1 and anti-Chk2 were used. 


\section{RESULTS AND DISCUSSION}

\section{Computational Analysis of the Binding between Anthocyanin and CDK5-p25}

On the basis of the experimental data, computational docking studies were performed to understand the possible binding site location and the best of conformation for binding of 2 anthocyanins to CDK5-p25 using binding free energy assessment. The binding energy of 50 models in docking is shown (Figure 1). Compared with the binding patterns, anthocyanins may bind to CDK5-p25 and was located in a region between the $\alpha$-domain and the $\beta$-domain, as depicted in Figure 1. the stabilizing effect contributed by anthocyanins on the appendant structure of CDK5-p25 may prevent the occurrence of domain swapping, so as to redirect CDK5-p25 away from the fibril-forming pathway and into forming nontoxic, unstructured and off-pathway aggregates. ${ }^{12}$

In our research, We speculated that two anthocyanins can inhibit the fibrillation of human Cytosolic Neutral betaGlycosylceramidase (Klotho-related Prote: CDK5-p25) complex. ${ }^{12,13}$ This observation is extremely importance as these two anthocyanins could protect the stability of Klotho and after interacting to avoid the occurrence of redox reaction, so as to achieve the role of anti-aging effect after interacting with protein.

\section{Effect of Anthocyanins on Bodyweight and Heart histology in Ageing Mice}

During 8 weeks of feeding, the dynamic changes of body weight were recorded and from Figure $2 \mathrm{~A}$ we can conclude that there was no significant difference, compared with brain index and liver index, the heart index also had no obvious difference in all groups mice. According to the haematoxylin-eosin staining, the pathological heart section displayed some necrotic heart cells in the model group, illustrating that ageing could bring about heart injury. Anthocyanins could significantly improve heart injury. In the treatment groups, the degree of heart fibrosis was decreased by anthocyanins in a dosedependent manner and anthocyanin treatments of $20 \mathrm{mg} / \mathrm{kg}$ and $40 \mathrm{mg} / \mathrm{kg}$ had a better effect of alleviating heart injury than $20 \mathrm{mg} / \mathrm{kg}$ TP (Figure 2B).

\section{Regulating Effects of Anthocyanins on the GSH- PX, T-SOD and MDA Content in Serum and Heart Tissue of Ageing Mice}

In order to judge ageing and health, the stability of the redox system is essential. From the Figure 3 we can see that the levels of GSH-PX and T-SOD of the control group were higher than the model group in both the serum and heart tissue. Anthocyanins obviously raised

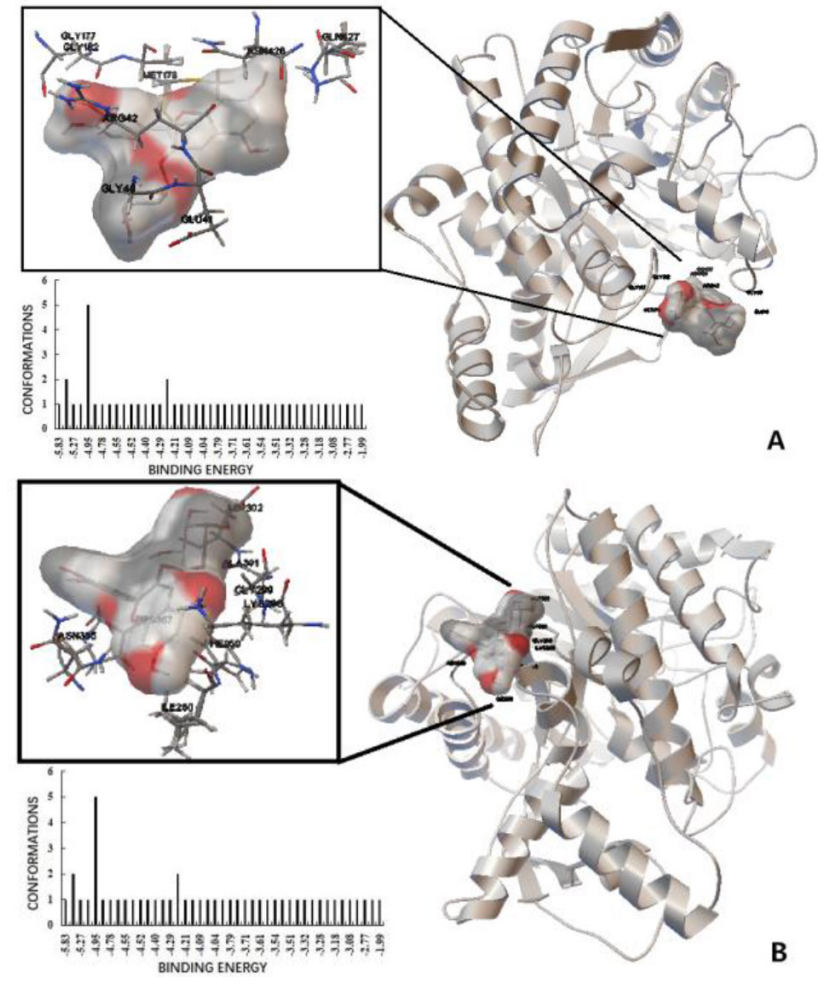

Figure 1: Use Autodock software to analyze 50 docking models of two anthocyanins and proteins. Panoramic view showing the binding mode between cyanidin 3-arabinoside (A), cyanidin 3-galactoside (B) and CDK5-p25. And the most conformations of binding energy are -4.95 .
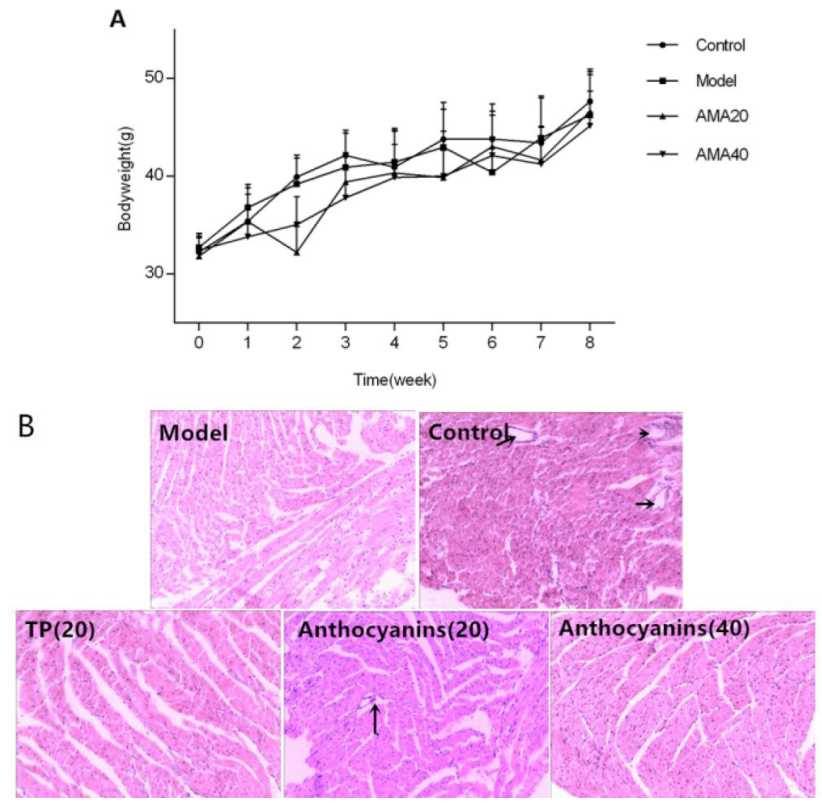

Figure 2: Effects of anthocyanins in Aronia melanocarpa on bodyweight and heart histology. A indicates the bodyweight; $B$ indicates the heart histology. 

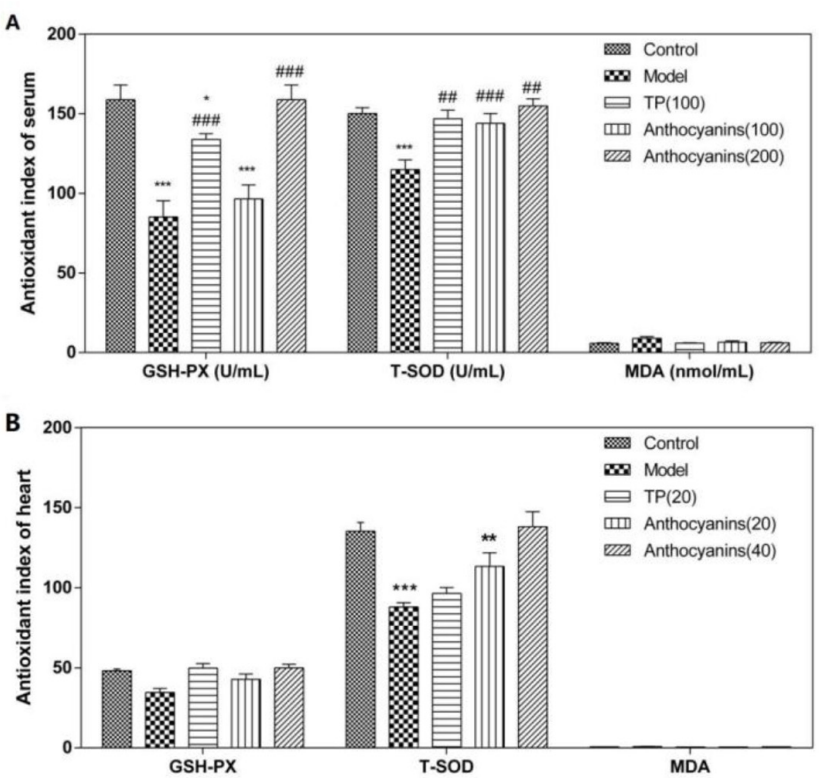

Figure 3: Effects of anthocyanins on the GSH-PX, T-SOD and MDA content. A indicates the GSH-PX, T-SOD and MDA content in serum; B indicates GSH-PX, T-SOD and MDA content in heart tissue. All datas represent the mean \pm SEM, ${ }^{*} p<0.05{ }^{* *} p<0.01{ }^{* \star *} p<0.001$ vs. Control, $\# p<0.05$ $\# \# p<0.01 \# \# \# p<0.001$ vs. Model.

the levels of GSH-PX and T-SOD in a dose-dependent manner in serum, compared with the model group and levels in the anthocyanins-40 group were significantly improved. However, the levels of MDA were lower in the control group than the model group and anthocyanins could decrease the levels of MDA. Furthermore, TP still had a positive effect on the results. We can conclude that anthocyanins in Aornia mealnocarpa can maintain the stability of the redox systemin in the heart.

\section{Regulating Effects of Anthocyanins on the DNA Damage Signalling Pathway}

DNA damage is a common feature of ageing cells and accelerates the ageing process. ${ }^{6,14}$ DNA damage responses are orchestrated by multiple signal transduction processes, among which the ATM-Chk2 and ATR-Chk1 pathways are key; these pathways are activated by DNA double-strand breaks (DSBs) and single-stranded DNA, respectively. ${ }^{5}$ During our research, the expression of ataxia telangiectasia mutated (ATM), ATM and Rad3related protein (ATR), Checkpoint kinase 1 (Chk1), checkpoint kinase 2 (Chk2) were all examined. From the Figure 4A, the expression levels of ATM and ATR were showed. The expression of ATM and ATR in the model group were increased. The expression of ATM was decreased by anthocyanins (20 and $40 \mathrm{mg} / \mathrm{kg}$ ) in a dose-dependent manner. Meanwhile the expression of ATR was higher in the $40 \mathrm{mg} / \mathrm{kg}$ anthocyanin group than in the $20 \mathrm{mg} / \mathrm{kg}$ anthocyanin group and was similar to that in the model group. The effects of the $20 \mathrm{mg} / \mathrm{kg}$
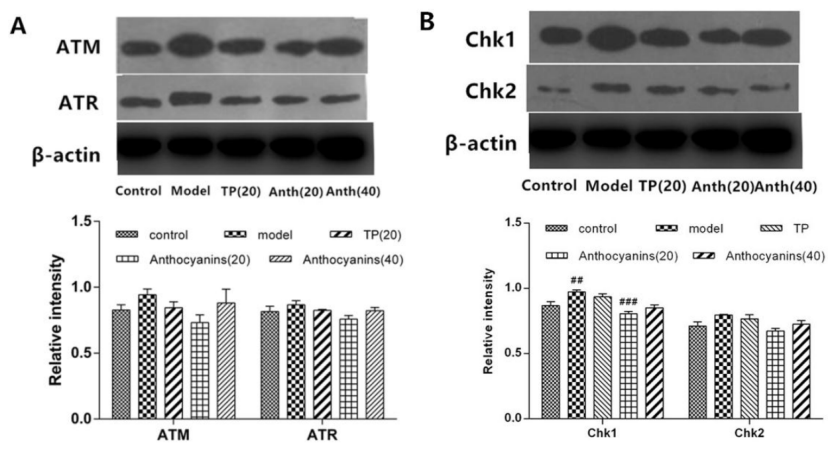

Figure 4: Regulating effects of anthocyanins on the DNA damage signalling pathway in the heart. (A) ATM and ATR; (B) Chk1 and Chk2. All datas represent the mean \pm SEM, ${ }^{*} p<0.05{ }^{* *} p<0.01{ }^{* * *} p<0.001$ vs. Control, $\# p<0.05$ $\# \# p<0.01 \# \# \#<0.001$ vs. Model.

TP treatment on ATR expression were more obvious than those of the anthocyanins treatments. However, the above results were not statistically significant. According to Figure 4B, the expression levels of Chk1 and Chk2 are shown. We can conclude that the expression of Chk1 was increased in the model group. Compared to the model group, the anthocyanins-20 group had decreased expression of $\mathrm{Chk} 1$ in the control group and TP also had a positive effect on Chk1 expression. In our research, there were no obvious changes in the expression of Chk2 between groups. And so far, It was also proved in our previous research that anthocyanins have a good inhibitory effect on brain ageing and nerve damage induced by DNA damage. ${ }^{15-17}$

We can conclude that Anthocyanin treatments were effective in slowing ageing-related degeneration of the heart. According to some previous research, anthocyanins can prevent fatty heart disease, heart fibrosis and other chronic heart diseases. ${ }^{18}$ In addition, anthocyanins have beneficial effects on the prevention and treatment of other diseases, such as cardiovascular diseases, cancer and neurodegenerative diseases. ${ }^{19-21}$

\section{CONCLUSION}

In conclusion, Aging is an integral part of the life process and is closely related to DNA damage. Based on the anti-brain and anti-liver aging effects of anthocyanins, the in-depth study of it delays the degenerative changes associated with heart aging. ${ }^{18}$ Our study aims to preliminarily elucidate the response to DNA damage caused by aging. The molecular mechanism of action provides a scientific theoretical basis for the development and utilization of anthocyanins in AMA. ${ }^{19-21}$

\section{ACKNOWLEDGEMENT}

This work was supported by the Science and Technology Government Department Fund in Liaoning province 
(Grant No. LYB201611) and the National Natural Science Foundation of China (Grant No.31701656).

\section{CONFLICT OF INTEREST}

The authors declare no conflict of interest.

\section{ABBREVIATIONS}

ATM: Ataxia telangiectasia mutated; ATR: ATM and Rad3-related protein; Chk1: Checkpoint kinase 1; Chk2: Checkpoint kinase 2; GSH-PX: Glutathione peroxidase; MDA: Malondialdehyde; SOD: Superoxide dismutase.

\section{Compliance with Ethical Standards Ethical approval}

This article does not contain any studies with human participants performed by any of the authors.

\section{REFERENCES}

1. Gregg $S Q$, Gutiérrez V, Robinson AR. A mouse model of accelerated liver aging caused by a defect in DNA repair. J Hepatology. 2012;55(2):609-21.

2. Houtkooper RH, Argmann C, Houten SM. The metabolic footprint of aging in mice. J SCI REP-UK. 2011;9:134.

3. Sheedfar F, Di BS, Koonen D. Liver diseases and aging: friends or foes?. J Aging Cell. 2013;12(6):950-4.

4. Paradis V, Youssef N, Dargere D. Replicative senescence in normal liver, chronic hepatitis $\mathrm{C}$ and hepatocellular carcinomas. Hum Pathol. 2001;32(3):327-32.

5. Rhind N, Russell P. Chk1 and Cds1: linchpins of the DNA damage and replication checkpoint pathways. J Cell Sci. 2000;113(22):3889-96.

\section{PICTORIAL ABSTRACT}

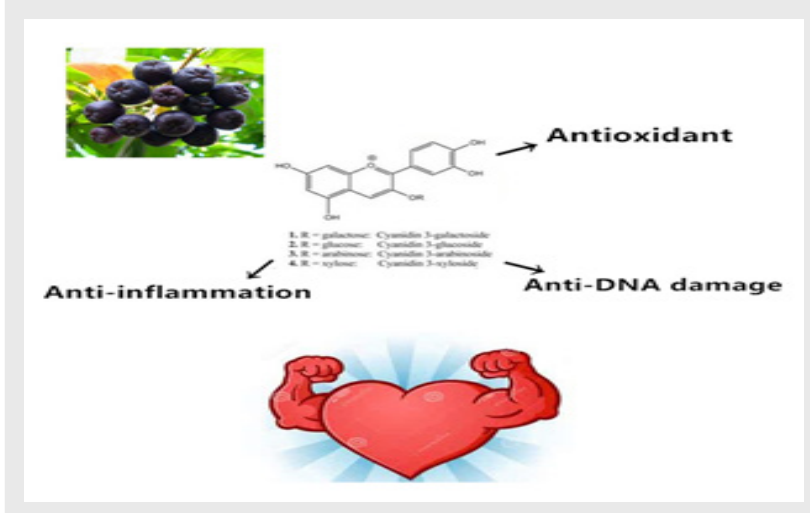

About Authors
6. Soares JP, Cortinhas A, Bento T. Aging and DNA damage in humans: a metaanalysis study. Aging. 2014;6(6):432-9.

7. Maynard S, Fang EF, Scheibye-Knudsen M. DNA Damage, DNA Repair, Aging and Neurodegeneration. J Csh Perspect Med. 2015;5(10).

8. Kulling S, Rawel H. Chokeberry (Aronia melanocarpa): A Review on the Characteristic Components and Potential Health Effects. Planta Med. 2008;74(13):1625-34.

9. Wei J, Zhang G, Zhang X, Gao J, Fan J, Zhou Z. Anthocyanin improving metabolic disorders in obese mice from Aornia melanocarpa. Indian J Pharm Educ. 2016;50(3):368-75.

10. Jie W, Guokun Z, Xiao Z. Anthocyanin Improving Metabolic Disorders in Obese Mice from Aornia melanocarpa. J Indian J Pharm Educ. 2016;50(3):368-75.

11. Jie W, Guokun Z, Xiao Z. Anthocyanins Delay Ageing-Related Degenerative Changes in the Liver. Plant Foods for Human Nutrition. 2017;72(4):1-7.

12. Hayashi $\mathrm{Y}$, Okino $\mathrm{N}$, Kakuta $\mathrm{Y}$, Klotho-related protein is a novel cytosolic neutral beta-glycosylceramidase. J Biol Chem. 2007;282(42):30889-900.

13. Hayashi $\mathrm{Y}$, Ito M. Klotho-Related Protein KLrP: Structure and Functions. Vitam Horm. 2016;101:1-16.

14. Sancar A, Lindseyboltz LA, Ünsalkaçmaz K. Molecular Mechanisms of Mammalian Dna Repair and the DNA Damage Checkpoints. Annu Rev Biochem. 2004;73(1):39-85.

15. Wei J, Zhang G, Zhang X. Anthocyanins from black chokeberry (Aronia melanocarpa Elliot) Delayed Aging-related Degenerative Changes of Brain. J Agr Food Chem. 2017;65(29):5973 -84.

16. Jiang Z, Chen C, Xie W, Anthocyanins attenuate alcohol-induced hepatic injury by inhibiting pro-inflammation signalling. Nat Prod Res. 2015;30(4):1-5.

17. Popović D, Đukić D, Katić V. Antioxidant and proapoptotic effects of anthocyanins from bilberry extract in rats exposed to hepatotoxic effects of carbon tetrachloride. Life Sci. 2016;157:168-77.

18. Min J, Yu SW, Baek SH. Neuroprotective effect of cyanidin-3-O-glucoside anthocyanin in mice with focal cerebral ischemia. Neurosci Lett. 2011;500(3):157-61.

19. Lin BW, Gong CC, Song HF. Effects of Anthocyanins on the prevention and treatment of cancer. Brit J Clin Pharmaco. 2017;174(11):1226-43.

20. Subash S, Essa MM, Al-Adawi S. Neuroprotective effects of berry fruits on neurodegenerative diseases. Neural Regen Res. 2014;9(16):1557-66.

21. Hügel HM, Jackson N, May B. Polyphenol protection and treatment of hypertension. Phytomedicine. 2016;23(2):220-31.

\section{Summary}

- The core of the aging process is DNA damage, a food additive called Anthocyanin which has shown high efficacy in preventing aging, Mice of Kunming were injected with D-galactose and they were given anthocyanins of 20 and $40 \mathrm{mg} / \mathrm{kg}$ as an intervention. The whole function and structure of heart the expression levels of genes involved in the DNA damage signalling pathway were evaluated through Western-blot analysis. Anthocyanins can be diffusely used to slow ageing-related deterioration of heart structure and function by inhibiting DNA damage in the field of health products.

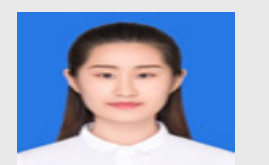

Ms.Dexin Xu, completed her master degree under Pro. Jie Wei in Life Sciences from Liaoning University in 2016, currently she is Pursuing Majors in Food Science.

Cite this article: Xu D, Yang J, Yu W, Wei J. Anthocyanins from Black Chokeberry Delayed Ageing-related Degenerative Changes in the Heart. Indian J of Pharmaceutical Education and Research. 2019;53(1):112-6. 